\title{
O uso de displays estereoscópicos em hipermídias
}

\author{
The use of stereoscopic displays in hipermedias
}

\author{
Gustavo E. Boehs \\ Universidade Federal de Santa Catarina, Brasil \\ gustavoeb@gmail.com \\ Marília M. Gonçalves \\ Universidade Federal de Santa Catarina, Brasil \\ c2mmg@hotmail.com \\ Alice T. Cybis Pereira \\ Universidade Federal de Santa Catarina, Brasil \\ acybis@gmail.com \\ Milton L. Horn Vieira \\ Universidade Federal de Santa Catarina, Brasil \\ milton@cce.ufsc.br
}

\section{RESUMO}

Com o recente aumento uso da tecnologia estereoscópica nas telas de cinema, alguns novos equipamentos eletrônicos passaram a incorporar esta tecnologia. No entanto, a natureza narrativa do cinema difere da natureza hipermidiática do conteúdo de equipamentos como computadores e celulares. O presente trabalho busca, portanto, responder a pergunta "Qual a relevância da tecnologia estereoscópica para as hipermídias e como futuros trabalhos de pesquisa podem vir a contribuir para a construção de conteúdo hipermidiático estereoscópico?" Para tanto foi feita uma revisão sistemática de literatura. Foram encontrados trabalhos nos contextos da Educação, Medicina, Comunicação e Entretenimento, Museologia e Interação Homem-Máquina. Em nenhum dos trabalhos, no entanto, a estereoscopia foi isolada como variável de pesquisa para avaliação do diferencial da mesma para os referidos campos.

Palavras-Chave: estereoscopia, hipermídia, hipermídia estereoscópica, imersão

\begin{abstract}
With recent increase the use of stereoscopy (popularly known as $3 d$ ) in cinema screens, some of most recent electronic equipment have built in such technology. Nevertheless the narrative nature of cinema differs from the hypermediatic nature of content provided through some of this equipment like computers and cell phones. Therefore the present work aims at answering the question "What is the relevance of stereoscopic technology for the hypermedia andh how may future researches contribute to hypermediatic stereoscopic content?" To address that question a systematic literature research was conducted. Works were found in the context of Education, Medicine, Entertainement and Comunication, Museology, and Human-Computer Interaction. However in none of this works stereoscopy was treated as an isolated variable.
\end{abstract}

KeyWords: stereoscopy, hypermedia, stereoscopic hypermedia, immersion 
BOEHS, Gustavo; GINÇALVES, Marilia; PEREIRA, Alice; VIEIRA, Milton

\section{INTRODUÇÃO}

Estereoscopia é uma tecnologia de reprodução de imagens que consiste na exibição simultânea de duas imagens. Tais imagens são capturadas em pontos diferentes do espaço, visando simular o comportamento do olho humano e, portanto, tornar a experiência daquele que as visualiza mais próxima de uma experiência real. Vulgarmente da-se o nome a este efeito de 3D, dado o aumento da sensação de profundidade por ele causado. No entanto a estereoscopia é apenas um de vários índices de profundidade reconhecidos pela percepção humana. Outros índices incluem, por exemplo: oclusão, espaçamento e tamanho dos objetos.

Além disso, vale ressaltar nesta introdução, que o termo 3D é usado para designar gráficos gerados em computação gráfica de geometrias tridimensionais, sem que estes gráficos sejam necessariamente estereoscópicos. Esta partilha de termos em áreas que se interrelacionam com freqüência pode gerar confusão e, portanto, neste trabalho designaremos imagens de geometrias tridimensionais geradas em computação gráfica como CG-3D. Nos eventos onde os autores designaram estereoscopia com seu nome popular (3D) o autor deste trabalho reservouse o direito de complementar com o prefixo "Estereo" afim de evitar confusões. Nestes casos o leitor encontrará a informação da segunte maneira Estereo-3D.

De qualquer modo, a estereoscopia não é tecnologia nova, sua criação precede a fotografia. Recentemente o grando númer de espectadores em filmes estereoscópicos tem impulsionado a implementação desta tecnologia em diversos artefatos eletrônicos. No entanto existe uma diferença entre a linguagem narrativa do cinema e as possibilidades hipermidiáticas e multimodais de alguns destes equipmantos eletrônicos, como celulares e computadores.

Este trabalho busca responder portanto a seguinte pergunta "Qual a relevância da tecnologia estereoscópica para as hipermídias e como futuros trabalhos de pesquisa podem vir a contribuir para a construção de conteúdo hipermidiático estereoscópico?".

Para tanto foi realizada uma pesquisa sistematica em diferentes bases de dados disponíveis na internet, com o seguinte objetivo: "Buscar pesquisas recentes, a respeito do uso da estereoscopia em hipermídias e mídias hipermidiáticas"

\section{MÉTODO}

A presente pesquisa fez uso de uma busca sistemática por publicações em bases de dados científicas disponíveis na internet. Inicialmente foram selecionados para a busca os termos "hipermidia" e "estereoscócipa" (em inglês). Foi consultada a ferramenta de periódicos da CAPES, através de uma busca simples dentro da área das "Ciências Sociais Aplicadas" que por sua vez indexa uma série de base de dados como ACM Library, Springer Link, SciELO, Science Direct, etc. Esta busca resultou em um total de 208 artigos.

No intuito de encontrar o maior número de trabalhos relevantes para esta pesquisa, dentro das limitações de tempo e escopo, foi feita uma segunda busca substituindo o termo "estereoscópica" por " $3 d$ ". Na linguagem popular o efeito da estereoscopia e os produtos que fazem uso desta tecnologia são designados com o termo " $3 d^{\prime \prime}$, dada a sensação de profundidade causada pelo mesmo. 0 termo $3 \mathrm{~d}$, no entanto, em grande número de publicações científicas, designa a reprodução de formas geométricas, sem qualquer implicação com o efeito estereoscópico. Foi verificado que um número consideravel das publicações encontradas com estes termos abordavam CG-3D e não Estereo-3D. Estes resultados foram descartados.

Dos 208 artigos encontrados o autor deste trabalho filtrou os trabalhos de acordo com a data de sua publicação. Foram mantidos apenas aqueles publicados a partir de 2009, resultando em um total de 45 artigos aqui analisados. Posteriormente através de uma criteriosa leitura dos resumos, introduções, metodologias e conclusões destes trabalhos foram descartados os 
que não tinham como objeto de pesquisa o uso de hipermídias estereoscópicas. Foi verificado que alguns destes trabalhos citavam os 2 termos pontualmente sem nunca relaciona-los. Restaram então para a análise durante o decorrer deste artigo um total de 8 publicações.

\section{RESULTADOS}

Quanto a origem dos trabalhos encontrados Singapura, Coreia do Sul, Italia, Finlândia, França, Japão apresentaram um artigo cada, e Israel apresentou dois artigos.

Quanto ao contexto dos objetos de pesquisa o autor identificou 5 grupos distintos. Os trabalhos que trataram das hipermídias e estereoscopia no contexto da educação, da medicína, da comunicação e entretenimento, da museologia e da interação homem-computador. A análise do trabalho dar-se a delimitada por estes contextos.

\subsection{Educação}

Nesta categoria foram encontrados três trabalhos: (1) "Visual Immersive Mathematics In 3d Web", (2) "Enhancement Of Spatial Thinking With Virtual Spaces 1.0" e (3) "The Synergetic Effect Of Learning Styles On The Interaction Between Virtual Environments And The Enhancement Of Spatial Thinking". Todos os trabalhos possuiam como objeto de estudo ambientes de realidade virtual, hipermidiáticos e multi-modais, onde o foco era o ensino de geometria espacial. Todos os trabalhos possuiam em comum o referencial teórico da corrente pedagógica do construtivismo, segundo a qual "humanos geram conhecimento e significado a partir de suas experiências" (LAI e SOURIN, 2011).

Vale salientar que os dois últimos trabalhos citados possuem o mesmo autor principal, Hanoch Hauptman, e utilizam em suas pesquisas o mesmo ambiente virtual "Virtual Spaces 1.0".

Lai e Sourin (2011), em "Visual Immersive Mathematics In 3d Web", afirmam que em suas pesquisas preliminares foram encontrados cerca de 40 experimentos de ambientes virtuais hipermidiáticos para o ensino de geometria especial. Dentre estes citam as características dos 8 experimentos que consideram mais relevantes. Entre eles dois se mostram relevantes dentro do contexto da presente pesquisa, a experiência de Nikolais et al. (2004, apud LAI e SOURIN 2011) e a de Cuixia et al. (2009, apud LAI e SOURIN 2011). A primeira se deu fazendo uso de um display estereoscópico e a segunda fez uso de um ambiente CAVE estereoscópico. CAVE ou Cave Automatic Virtual Envoiroment são salas capazes de exibir imagens estereoscópicas, ou não, em todas suas paredes e piso, imergindo o espectador completamente no espaço virtual.

Nos dois casos Lai e Sourin havaliam que a noção de profundidade trazida pela estereoscopia trouxe maior riqueza para a visualização dos conteudos propóstos.

Hauptman, em "Enhancement of spatial thinking with Virtual Spaces 1.0" (2010), no capítulo "Trabalhos Relacionados" verifica que a maioria das pesquisas no campo do ensino da geometria espacial através da realidade virtual não fazem uso da estereoscopia. Segundo o autor:

\footnotetext{
“a melhor visualização do efeito tridimensional [fazendo uso da estereoscopia] foca a atenção do estudante sobre os signos que o ajudam a extrair significado da informação que lhe é ofertada, portanto, ajudando-o a superar as dificuldades do estudo da geometria tridimensional". (HAUPTMAN, 2010, p. 126)
}

Apesar de Lai e Sourin apontarem a estereoscopia como um destaque em alguns dos trabalhos encontrados em suas pesquisas preliminares, o ambiente virtual proposto e estudado por estes autores não fazia uso da estereoscopia. Não foi por estes citado um motivo específico para tal omissão, assim como não foi feita nenhuma referência ao uso desta tecnologia em 
BOEHS, Gustavo; GINÇALVES, Marilia; PEREIRA, Alice; VIEIRA, Milton

trabalhos futuros. Já no trabalho de Hanoch o uso da estereoscopia foi citado como o grande diferencial entre este e os demais estudos que o precederam, nas palavras do autor:

“Em geral a grande diferença entre o Virtual Spaces 1.0 e outros softwares que focam em geometria espacial como, por exemplo, o 3DMath, ou programas que avançam o conhecimento de conceitos geométricos como 3D-lab e HyperGami, é que os objetos destes ambientes aparecem em telas planas, bidimensionais, enquanto no Virtual Spaces 1.0 os objetos são verdadeiramente tridimensionais." (HAUPTMAN, 2010, p. 127)

Hauptman verifica ainda a eficiência da ferramenta proposta através de um experimento feito com 194 alunos de segundo grau. Através da análise dos dados coletados o autor pode concluir que o uso da ferramenta proposta aumentou de maneira considerável o entendimento dos estudantes sobre a geometria espacial, quando comparado com métodos tradicionais de ensino. $O$ autor verificou ainda que quando combinados os dois métodos de ensino, já citados, os resultados em tarefas básicas não eram melhores do que quando foi utilizado apenas o método de realidade virtual. No entanto em tarefas complexas o grupo que usou a combinação dos dois métodos de ensino teve os resultados mais expressivos.

Em "The Synergetic Effect Of Learning Styles On The Interaction Between Virtual Environments And The Enhancement Of Spatial Thinking" Haupman e Cohen (2011) verificam a eficiência do "Virtual Spaces 1.0" em grupos com diferentes "estilos de aprendizado", posteriormente sub-categorizados em "estilos de aprendizagem modais" e "estilos de aprendizagem por personalidade".

A taxonomia de estilos de aprendizagem modais é baseada no modelo de Miller (2000, apud LAI e SOURIN 2011) e contém 3 categorias:

- Aprendizado visual

- Aprendizado auditivo

- Aprendizado cinético

- A taxonomia de estilos de aprendizagem por personalidade é derivada do modelo de Myers-Briggs e contém X categorias:

- Extrovertidos (E)

- Introvertidos (I)

- Intuitivos (N)

- Sensoriais (S)

- Emocionais (F)

- Racionalistas (T)

Quanto aos estilos de aprendizagem modais os resultados mostraram que, diferentemente do esperado, não houve diferença significativa entre os 3 grupos. Apesar do grupo de aprendizado visual ter obtido melhores resultados a diferença para com os outros 2 grupos foi considerada insignificante pelos autores do trabalho. Foi levantada pelos autores a hipótese de que a natureza visual do ambiente virtual pode estar tornando "complexos conceitos abstratos mais compreensivos de maneira a superar as inibições de diferentes estilos" (HAUPTMAN e COHEN, 2011, p.2115).

Quanto aos estilos de aprendizagem por personalidade foi verificado que estudantes do estilo Sensorial superam os estudantes Intuitivos apenas quando o aprendizado dase unicamente através do método de realidade virtual. Quando combinado com métodos tradicionais foi verificado que o grupo Sensorial não apresentou resultados significativamente 
superiores a outros grupos, como era esperado pelos pesquisadores. A hipótese levantada pelos mesmos, é que dada a natureza verbal dos métodos de ensino tradicionais a natureza primariamente sensorial dos estudantes pode ter sido afetada negativamente.

Por fim o trabalho concluí que apesar de terem sido verificadas diferenças entre os estilos de aprendizado dentro dos ambientes virtuais, a mesma não foi suficiente para inferir uma resposta inequívoca a pergunta de pesquisa: "Os estilos de aprendizagem são relevantes no contexto dos ambientes virtuais?". O trabalho concluiu também que o uso de ambientes virtuais é um auxílio importante para estudantes com perfil sensorial e estudantes com modalidade cinética.

\subsection{Medicina}

Nesta categora é abordado o artigo: "A System for the Acquisition, Interactive Exploration and Annotation of Stereoscopic Images" da autoria de Benzeroual, et al. (2009). Tal artigo estudou o caso da criação de um sistema para a aquisição, exploração e exibição de imagens para o estudo e avaliação de casos dermatológicos.

Da etapa de aquisição vale ressaltar que foi usada uma técnica estereoscópica para a mensuração de profundidade das imagens. Dito isso as especificidades técnicas e metodológicas desta estão fora do escopo deste trabalho, que lida apenas com os displays estereoscópicos e não a geração ou captação destas imagens.

Da etapa de exploração o trabalho explicita que exitem uma série de pesquisas diferentes a respeito deste assunto, que por questões de escopo não foram abordadas. Dito isso dentro do sistema descrito o usuário poderia fazer anotações sobre as imagens capturadas em forma de texto ou áudio, e ainda escolher a ordem de exibição e tempo das imagens e anotações.

Quanto a exibição, as imagens foram expostas em displays com dois tipos de tecnologia estereoscópica diferentes, sendo elas ativa (Onde os óculos utilzados pelo espectador são confexionados em cristal liquido e bloqueiam a visualização hora de um olho, hora de outro, em alta frequencia e em sincronia com o display utilizado) e passiva (Onde os óculos utilzados pelo espectador são confexionados em um material polarizado que filtra duas imagens exibidas ao mesmo tempo, uma para cada olho). No entanto, como não era objetivo da pesquisa havaliar a diferença entre estas duas tecnologias, nenhum dado sobre a reação do usuário à estas foi coletado.

Como forma de avaliar a validade do sistema proposto foram capturadas imagens de 18 pacientes diferentes, entre 20 e 80 anos. $O$ autor verificou que as ferramentas propostas funcionaram e ainda que as "possibilidades trazidas pela ferramenta melhoraram o diagnóstico de diferentes sintomas da pele tornando a identificação de informações específicas mais fácil que em fotografias normais (Benzeroual, et al., 2009). No entanto o autor não traz mais detalhes sobre os métodos utilizados e dados coletados para verificar estas afirmações.

\subsection{Comunicação e Entretenimento}

Nesta categoria foram encontrados dois artigos: (1) "iBaton Conducting Virtual Concerts Using Smartphones" e (2) "3DTV as a Social Platform for Communication and Interaction". Ambos os trabalhos lidam com objetos onde as funções predominantes são ou do entretenimento ou da comunicação.

O primeiro artigo analisado "iBaton Conducting Virtual Concerts Using Smartphones" da autoria de Ranaweera, et al. (2012) é um estudo de caso que descreve a criação de um ambiente virtual que simula a situação de uma orquestra, onde o sujeito torna-se o maestro. Constitue essa descrição as decisões técnicas tomadas pelos autores e as razões práticas das mesmas. No entanto, as razões conceituais que fundamentam tais escolhas não são profundamente explicitadas.

No que tange a relevância do artigo para o presente trabalho foi escolhido um display estereoscópico para a exibição do conteúdo gerado. Os autores explicam que a estereoscopia é: "uma técnica para realçar a ilusão de de profundidade em uma imagem" (RANAWEERA, COHEN e ENDO, 2012). No entanto, não há qualquer outra informação a respeito da razão da escolha desta técnica, do seu impacto durante a implementação do projeto bem como da sua influência sobre o usuário e o 
objeto de pesquisa.

O segundo artigo aqui analisado "3DTV as a Social Platform for Communication and Interaction" da autoria de Shin (2012) é uma pesquisa que busca tornar evidentes elementos que podem ser relevantes na aceitação tecnologica da TV [Estereo-]3D no contexto doméstico. No contexto do presente trabalho este artigo se mostra relevante na medida que (1) Busca comprender fatores que influenicam a aceitação de um artefato de tecnologia estereoscópica por parte de um público e (2) Não trata a TV [Estereo-]3D como mero reprodutor de conteúdo de vídeo, mas como plataforma multimidática, multimodal e com capacidades socio-interativas.

Para execução da citada análise a pesquisa toma como princípio o método TAM (Technology Acceptance Model, ou em portugês, Modelo de Aceitação Tecnológica). Dada algumas limitações do modelo, proprimamente definidas no artigo, o autor opta por adaptar a TAM para a ocasião deste estudo. Para isto o mesmo traz elementos da TRA (Theory of Reasoned Action, ou em português, Teoria do Racionamento de Ações) e de pesquisas anteriores que tiveram a TV [Estereo-]3D como objeto de estudo.

Por fim os modelos teóricos escolhidos pelo autor, bem como as hipóteses levantadas por ele podem ser descritos da seguinte maneira. A qualidade percebida influencia a utilidade percebida, assim como a qualidade de conteúdo percebida influencia a satisfação percebida e todos estes elementos juntos influenciam a atitude do usuário quanto ao sistema. A atitude por sua vez, junto com o flow e a presença social influenciam a intenção do usuário em adotar o sistema, logo a intenção do usuário implica no seu uso. Este raciocínio foi esquematizado pelo autor da seguinte maneira:

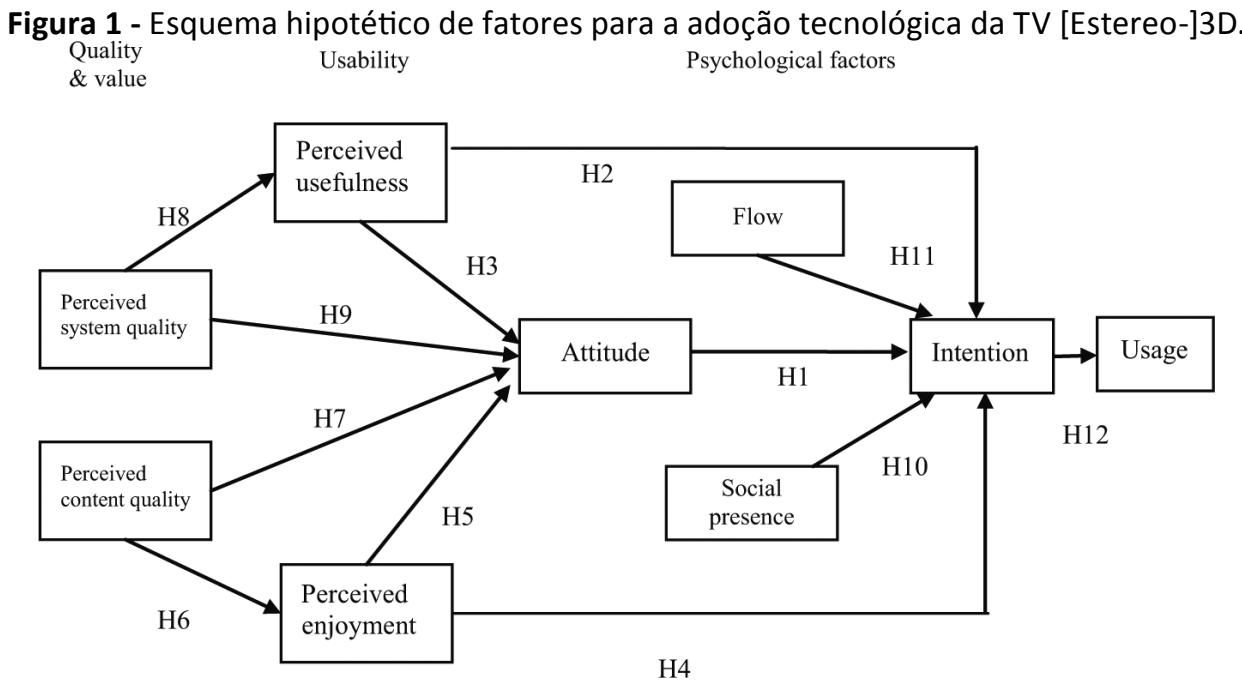

Fonte: SHIN, 2012.

Para o melhor entendimento do que foi proposto é importante explicitar as definições feitas pelo autor sobre conceitos incomuns ou discordantes do censo comum:

- Utilidade percebida: "grau a que uma pessoa acredita que usar um sistema em particular pode realçar sua produtividade" segundo Davis (1989, apud. SHIN, 2012). Shin (2012) alerta que o significado de produtividade varia entre os contextos coletivo e pessoal.

- Satisfação percebida: motivação intrínseca é definida pela capacidade do usuário em "perceber uma ação como satisfatória em seu próprio fim à parte do valor instrumental da tecnologia" segundo Davis (1989) citado em (SHIN, 2012).

- Presença social: "sentimento de estar em companhia de outro(s), e ilusão perceptiva de não mediação" (Shin, 2012).

- Flow: experiência de estar "completamente imerso, completamente focado e motivado na ação" segundo Csikszentmihalyi (1977) citado em Shin (2012). 
Fim elaborado então um questionário que pudesse validar ou refutar as hipóteses levantadas. O mesmo foi distribuido e preenchido através da internet por 229 participantes.

Os resultados obtidos com a coleta e análise dos dados permitiram ao autor confirmar algumas hipótes e refutar outras, chegando as seguintes conclusões: (1) A presença social é influenciada e influencia mutuamente o flow. Por sua vez a presença social influencia unidirecionalmente a utilidade percebida, a satisfação percebida, a qualidade percebida e a qualidade de uso percebida, bem como todas as interações entre estas (previamente descritas). (2) Diferente da hipótese inicial foi verificado que a atitude positiva quanto a tecnologia não tem a forte influência esperada sobre a intenção de adoção, segundo o autor esta incosistência poderia ser explicada pela necessidade dos usuários em confirmar a qualidade e disponibilidade da tecnologia.

\subsection{Museologia}

Nesta categoria foi encontrado o artigo "From 3D Reconstruction To Virtual Reality: A Complete Methodology For Digital Archaeological Exhibition" da autoria de Bruno et al. (2010). O referido artigo é o estudo de caso da construção de um ambiente virtual, dentro do qual foram inseridos artefatos arquelógicos virtuais. Foram abordadas mais especificamente as etapas do escaneamento dos artefatos e construção dos modelos em CG-3D, desenvolvimento do software de visualização do conteúdo criado, interface deste mesmo software e a exibição do conteúdo para um determinado público.

Dentro do contexto da presente pesquisa este trabalho se mostrou relevante em dois momentos. (1) Na escolha do uso da estereoscopia devido a "possibilidade de o usuário explorar todos os detalhes do objeto" (BRUNO, et al., 2010) e (2) na verificação através de comentários ocasionais dos espectadores que, segundo o autor, mostravam interesse nas qualidade estereoscópica das imagens.

É importante ressaltar os 2 casos supracitados, apesar de indicativos de um fenômeno, não são confirmações efetivas do tal. Não houve, por parte do autor, a preocupação em explicitar uma estrutura metodologia sistemática para a escolha das tecnologias de exibição utilizadas. Ou seja não foram explicitadas, em um primeiro momento, fortes evidências de que o uso da estereoscopia teria um impacto positivo na experiência dos usuários. Da mesma forma não houver rigor metodológico, nem dados estatísticos que comprovem, de fato, um interesse maior dos espectadores sobre as imagens estereoscópicas do que sobre imagens monoscópicas.

\subsection{Interação Homem-Máquina}

Nesta categoria abordaremos o artigo encontrado cuja o objeto de estudo englobou características da interação homem-máquina no contexto dos ambientes virtuais hipermidiáticos. $\mathrm{O}$ artigo aqui abordado, "The Movement Patterns and The Experiential Components of Virtual Environments" (SARKELA, et al., 2009) não demonstraram comprometimento com um contexto de aplicação específico. $O$ foco está na maneira como os seres humanos percebem e interagem o artefato em questão.

A pergunta de pesquisa do citado trabalho é a seguinte: como avaliar a movimentação do sujeito dentro de ambientes virtuais e como comparar esta informação com outros componentes da experiência nestes artefatos? Segundo o autor este estudo se justifica, pois foi anteriormente verificado por outros trabalhos que enquanto a movimentação nos ambientes virtuais está "adimitidamente esta longe do ato de andar, foi mostrado que as estratégias básicas the movimentação em ambientes virtuais bem estruturados são similares as estratégias utilizadas em ambientes naturais" (Zacharias, 2006, apud. SARKELA, et al., 2009, p. 788).

$\mathrm{Na}$ ausência de métodos estruturados para a modelagem dos dados a serem coletados o autor propõe uma ferramenta de modelagem baseada no conceito da Entropia da Informação, o qual pressupõe que "entropia mede a quantidade de informação contida em uma fonte de dados" (Weaver, 1963, cited in Sarkela, et al., 2009, p.788), considerando como fonte de dado qualquer signo, e considerando que "quanto mais uma informação se repete menor a entreopia" (SARKELA, et al., 2009) sendo o inverso também verdadeiro.

Além disso o autor buscou outros componentes da experiência do usuário nos ambientes de realidade virtual que já possuíam uma modelagem definida e amplamente utilizada para descrição 
e mensuração destes fenômenos. Assim, o autor trouxe para o estudo o conceito da percepção de presença e percepção de flow. Segundo Lombard e Ditton (1997) citados em (SARKELA, et al., 2009) a presença, no contexto da realidade virtual, pode ser definida como a "ilusão perceptiva da não mediação". Enquanto Csikszentmihalyi (1975) citado em (Sarkela, et al., 2009) define que flow é "estado psicológico de concentração e divertimento".

Para o contexto do presente trabalho o artigo "The Movement Patterns and The Experiential Components of Virtual Environments" se faz relevante pois todas as experiências foram conduzidas em um ambiente CAVE Estereoscópico. De modo que os índices de profundidade advindos da estereoscopia sensibilizaram os sujeitos de pesquisa e influenciaram os resultados. No entanto, é importante salientar que o referido artigo não isolou a estereoscopia como uma das variáveis controladas para o experimento, tornando impossível determinar qual o papel específico desta na percepção de presença, no flow e na movimentação dos sujeitos de pesquisa. De qualquer modo dada a referida influencia da estereoscopia sobre os resultados, faz-se necessário aqui explicitar estes últimos.

Os autores do artigo dividiriam os participantes, de acordo com os resultados obtidos quanto a sua movimentação, em 4 grupos. Aqueles que movimentavam-se de maneira (1) fluída, os que movimentavam-se de maneira (2) fluída com intervalos de estagnação, os que se movimentavam de maneira (3) descontrolada e os que ficaram a maior parte do tempo (4) estagnados. Ainda segundo os autores todos os sujeitos conseguiram executar todas as tarefas propostas, de modo que, a quantidade da movimentação não estava diretamente ligada a qualidade da mesma. Quanto a eficiência dos grupos na execução das tarefas a ordem dos grupos (do mais eficiente para o menos eficiente) foi 1, 4, 2, 3 .

Foi verificado que a eficiência dos sujeitos e sua percepção de presença estavam relacionados, embora a variação dos dados não permita uma afirmação vigorosa neste sentido. Foi verificado fenômeno similar quando confrontadas a eficiência e o flow. Uma pergunta possível na sugestão do presente autor é, "qual a relevância do efeito estereoscópico na percepção de presença e no flow" e indiretamente, "qual a relevância do efeito estereoscópico na movimentação do sujeito dentro do ambiente virtual".

\section{CONSIDERAÇÕES FINAIS E TRABALHOS FUTUROS}

O presente trabalho obteve sucesso em seu objetivo de "buscar pesquisas recentes, a respeito do uso da estereoscopia em hipermídias e mídias hipermidiáticas". Quanto a busca dos artigos ficou claro que são poucos os artigos que abordam hipermídias estereoscópicas de fato. Dos 45 artigos encontrados dentro dos critérios definidos apenas 8 deles realmente tinam como objeto mídias concomitantemente mídias hipermidiáticas e estereoscópicas. 0 restante apenas citava a existência da estereoscopia sem, no entanto, tomala como objeto de pesquisa.

Quanto aos artigos aqui expostos foi verificado que a aplicação de hipermídias estereoscópicas tem se dado em diferentes contextos. Sendo o mais abordado, dentro do material encontrado a educação. Dentro desta área, especificamente, a matemática e a geometria foram os únicos contextos abordados. Futuros trabalhos poderiam então verificar se ambientes hipermidiáticos estereoscópicos podem ou não trazer benefícios para o ensino de outras disciplinas.

Foi verificado também que em nenhum dos textos a escolha da estereoscopia foi embasada em trabalhos que comprovassem estatisticamente sua eficácia dentro do contexto abordado. Ou ainda trabalhos que utilizassem a presença da estereoscopia como variável de controle para posteriormente inferir esta relação eficaz. Para este autor, este é um tema chave para trabalhos futuros, visto que são resultados que podem impactar um grande número de pesquisas.

\section{REFERÊNCIAS}

LAI, D. e SOURIN, A., 2011. Visual immersive mathematics in 3D web. New York, ACM.

HAUPTMAN, H., 2010. Enhancement of spatial thinking with Virtual Spaces 1.0. Computers \& Education, 54(1), pp. 123-135. 
HAUPTMAN, H. e COHEN, A., 2011. The Synergetic Effect of Learning Styles on the Interaction between Virtual Environments and the Enhancement of Spatial Thinking. Computers \& Education, 57(3), pp. 2106-2117.

BENZEROUAL, K., HAOUACH, M., GUINOT, C. e VENTURINI, G., 2009. A System for the Acquisition, Interactive Exploration and Annotation of Stereoscopic Images. Berlin, Heidelberg.

RANAWEERA, R., Cohen, M. e ENDO, S., 2012. iBaton: conducting virtual concerts using smartphones. New York, ACM.

SHIN, D.-H., 2012. 3DTV as a social platform for communication and interaction. Information Technology \& People, 25(1), pp. 50-88.

BRUNO, F. et al., 2010. From 3D reconstruction to virtual reality: A complete methodology for digital archaeological exhibition. Journal of Cultural Heritage, 11(1), pp. 42-49.

SÄRKELÄ, H. et al., 2009. The movement patterns and the experiential components of virtual environments. International Journal of Human-Computer Studies, 67(9), pp. 787-799. 